\title{
Adhesión Bacteriana en Hilo de Sutura de Seda Negra y Nylon Post Extracción Dental Simple: Ensayo Clínico Controlado
}

\author{
Bacterial Adhesion in Black Silk Suture Thread and Nylon \\ Post Simple Dental Extraction: Controlled Clinical Trial
}

\author{
Angel S. Asmat-Abanto ${ }^{1,2} ;$ Alexandra F. Ávila-Rodríguez² \& Elva Mejía-Delgado ${ }^{3}$
}

\begin{abstract}
ASMAT-ABANTO, A. S.; ÁVILA-RODRÍGUEZ, A. F. \& MEJÍA-DELGADO, E. Adhesión bacteriana en hilo de sutura de seda negra y nylon post extracción dental simple: ensayo clínico controlado. Int. J. Odontostomat., 13(2):132-136, 2019.

RESUMEN: El objetivo de este estudio fue comparar los hilos de sutura de seda negra y la sutura nylon con respecto a la adhesión bacteriana post extracción dental simple de piezas posteriores. El presente trabajo se desarrolló en la Clínica Odontológica y el Laboratorio de Microbiología de la Unidad de Ciencias Biomédicas y Biotecnología de la Universidad Privada Antenor Orrego (Trujillo, Perú), entre agosto y setiembre de 2014. Se trabajó con 19 unidades de muestreo para hilo de seda negra y 19 para nylon. El procedimiento de exodoncia para todos los pacientes fue estandarizado. Luego de la extracción, se procedió a suturar la herida quirúrgica, colocando dos puntos circunferenciales, uno con cada tipo de hilo. A los 7 días, los hilos fueron retirados y sometidos a análisis microbiológico. La adhesión bacteriana de los hilos de sutura seda negra y nylon fue comparada empleando el test de Wilcoxon. Además la adhesión bacteriana de cada hilo fue comparada con la adhesión en hilos estériles (control) empleando el test de Mann-Whitney para una $p<0,005$. Al comparar la adhesión bacteriana de ambos hilos, se encontró diferencia entre ellos $(p=0,027)$, correspondiendo mayor adhesión al hilo de nylon. La adhesión bacteriana fue mayor en el hilo de sutura de nylon comparado con el hilo de sutura de seda negra.
\end{abstract}

PALABRAS CLAVE: suturas, extracción dental, adhesión bacteriana.

\section{INTRODUCCIÓN}

En la cavidad oral existen más de 700 especies bacterianas (Zijnge et al., 2010; Venema et al., 2011) y su biología se centra en su ciclo vital e interacciones con el medio ambiente (Nazar, 2007).

La adherencia de los microorganismos se da mediante las adhesinas que se unen a las proteínas salivales, siendo la fijación entre los microorganismos una condición necesaria para el desarrollo de los biofilms (Bin AIShaibah et al., 2012).

En la mucosa oral, la sutura quirúrgica produce una respuesta tisular (Edmiston et al., 2006) en donde la colonización microbiana compite con la integración de las células tisulares en los biomateriales (Lüdecke et al., 2014).
Las suturas contaminadas son un riesgo para cualquier cirugía (Cruz et al., 2013), presentando un papel importante en la cicatrización de las heridas (Banche et al., 2007; Burkhardt et al., 2008; Ozkan et al., 2009). Por tanto, la elección de un buen material de sutura optimiza el resultado quirúrgico (Ghoreishian et al., 2009; Altiparmak \& Uckan, 2013).

Actualmente se utilizan diferentes tipos de suturas sintéticas en cirugía oral (Ghoreishian et al.; Israr \& Stassen, 2013), entre ellas, la seda y el nylon, ambos no-absorbibles.

La seda quirúrgica es multifilamento de origen orgánico con facilidad de manejo (Armas Moredo et al., 2009; Pons-Vicente et al., 2011), ampliamente uti-

\footnotetext{
${ }^{1}$ Escuela de Postgrado de la Universidad Nacional de Trujillo, Perú.

${ }^{2}$ Escuela Profesional de Estomatología de la Universidad Privada Antenor Orrego Trujillo, Perú.

${ }^{3}$ Facultad de Medicina Humana de la Universidad Privada Antenor Orrego, Trujillo, Perú.
} 
lizada para diferentes procedimientos (Pons-Vicente et al.). Las suturas de nylon permiten una buena manipulación intraoperatoria (Wong et al., 2007) y su resistencia mecánica es estable (Nozaki et al., 2012).

Cuando el material de sutura se contamina, los mecanismos de descontaminación de la herida se vuelven ineficaces debido a la adhesión de los microorganismos al sustrato (Cruz et al.). Banche et al. sugiere que las bacterias se adhieren con diferente afinidad a los distintos tipos de materiales de sutura; encontrándose mayor cantidad de bacterias en las suturas no reabsorbibles que en las absorbibles.

Por lo anteriormente expuesto, se realizó el presente trabajo que tiene como objetivo comparar los hilos de sutura de seda negra y la sutura nylon con respecto a la adhesión bacteriana post extracción dental simple de piezas posteriores, como aporte para la elección del material de sutura, basado en la evidencia científica.

\section{MATERIAL Y MÉTODO}

El presente ensayo clínico se desarrolló entre agosto y setiembre de 2014 en la Clínica Estomatológica y el Laboratorio de Microbiología de la Unidad de Ciencias Biomédicas y Biotecnología de la Universidad Privada Antenor Orrego (Trujillo - Perú). Para determinar el tamaño muestral se usó la fórmula que corresponde a comparación de medias de grupos pareados, donde $\mathrm{Za}=1,645, \mathrm{Zb}=1,282, \mathrm{~m} 1=21,1$ unidades formadoras de colonias (UFC) (media de la adhesión con hilo de seda, estimada mediante muestra piloto), $\mu 2=28,1$ UFC (media de la adhesión con hilo de nylon, estimada mediante muestra piloto) y $s=10,274$ (desviación estándar de la diferencia de adhesión entre el hilo de seda con el hilo nylon, estimada mediante prueba piloto), resultando 19 especímenes para seda y 19 para nylon.

Se realizó una prueba piloto y la calibración con la colaboración de una microbióloga, docente universitaria investigadora con 20 años de experiencia, respetando el mismo protocolo clínico y microbiológico, donde se midió la adhesión microbiana en UFC, empleando un total de 10 suturas con hilo de seda negra y 10 suturas con hilo de nylon. La prueba estadística para la calibración fue el coeficiente de correlación intraclase, cuyos resultados fueron 0,990 interevaluador y 0,985 intraevaluador.
Se eligieron para el estudio los pacientes ASA I de 18 a 50 años de edad, con indicación de extracción dental simple de pieza posterior donde sea necesaria la colocación de 2 puntos de sutura circunferencial. Se excluyeron a los pacientes que no aceptaron participar, los alérgicos a materiales de sutura utilizados y con incapacidad intelectual para participar en el estudio.

Una vez iniciado el estudio, la unidad fue eliminada si el hilo de sutura se perdió antes de la cita control para retiro de puntos, si el paciente no regresó a la cita para retiro de puntos y si se presentaron complicaciones en la herida quirúrgica derivada de la exodoncia antes de la cita de retiro de puntos.

Para la ejecución, se contó con la aprobación de la Escuela de Estomatología y de la Facultad de Medicina Humana de la Universidad Privada Antenor Orrego, considerando los principios éticos de la Declaración de Helsinki y de la Ley General de Salud del Perú (Ley N ${ }^{\circ}$ 26842).

Mediante la historia clínica se seleccionaron a los pacientes que cumplían con los criterios mencionados. De aceptar voluntariamente, estos firmaron el consentimiento informado antes del procedimiento.

Se siguió el mismo procedimiento de extracción dental simple usando como guía lo indicado por Gay \& Berini (2006) para todos los pacientes. Luego de la extracción, se procedió a suturar la herida quirúrgica, colocando dos puntos circunferenciales, uno de cada tipo de hilo, con $10 \mathrm{~mm}$ de distancia aproximada entre ellos.

Las indicaciones postquirúrgicas fueron las mismas para todos los pacientes, con la certeza que el paciente comprendió y manifestó su voluntad de colaboración con el estudio.

Se usaron en total 19 hilos de seda negra trenzado multifilamento 3-0 de Cirugía Peruana (con aguja 3/8 círculo punta reverso cortante, número de Lote: 1120833) y 19 hilos de sutura nylon monofilamento $3 / 0$ de Cirugía Peruana (con aguja 3/8 círculo punta reverso cortante; con el número de lote: 1041524). Para el control se usaron hilos estériles de cada tipo.

Cumplidos los siete días después de la extracción dental, se realizó el retiro de las suturas, siguiendo el mismo procedimiento para todos los pacientes, sujetando un extremo con una pinza estéril y cortando el hilo a ras de la mucosa. De esta manera, no se dejaron detritus al interior de los tejidos al sacar el cabo 
cortado. Estos fueron comparados con los hilos de sutura control.

Cada hilo de sutura se colocó en su respectivo tubo de ensayo, preparado con $1 \mathrm{ml}$ de medio Tioglicolato. Luego, en el laboratorio, estos tubos fueron centrifugados a $3,00 \mathrm{rpm}$ durante cinco minutos. Se tomó $0,1 \mathrm{ml}$ del sobrenadante de la muestra centrifugada el cual fue sembrado en placas petri con el medio cultivo de Tripticasa Soya Agar (TSA). Las placas Petri se colocaron en condiciones de microanaerobiosis, en estufa a $37^{\circ} \mathrm{C}$ por 24 horas.

Se contabilizaron las UFC en las placas Petri y se registraron en una ficha elaborada específicamente para la investigación.

Los datos fueron presentados en tablas con medias y desviaciones estándar. La adhesión bacteriana de los hilos de sutura seda negra y nylon fue comparada empleando el test de Wilcoxon. Además la adhesión bacteriana de cada hilo de sutura con el control fue realizado empleando el test de MannWhitney para una $p<0,005$.

\section{RESULTADOS}

La investigación incluyó un total de 19 pacientes, en los que se colocaron dos puntos de sutura (una de cada tipo) en la herida quirúrgica. Las muestras fueron cultivadas en placas Petri. Fueron eliminados 7 pacientes del estudio por presentar los criterios de eliminación establecidos.

Al comparar la adhesión bacteriana de ambos hilos, se encontró diferencia entre ellos ( $p=0,027)$, correspondiendo mayor adhesión al hilo de nylon (Tabla I).

En las Tabla II y III, se puede apreciar que tanto el hilo de seda negra como el hilo de nylon, presentaron mayor adhesión bacteriana que sus correspondientes controles ( $p=0,000$ para ambos).

Tabla I. Comparación de los hilos de seda negra y nylon con respecto a la adhesión bacteriana post extracción dental simple.

\begin{tabular}{lll}
\hline & Seda & Nylon \\
\hline Media & 87,5 & 99,2 \\
D. S. & 206,0 & 236,1 \\
Wilcoxon: Z & 1,932 & \\
p & 0,027 & \\
\hline
\end{tabular}

Tabla II. Adhesión bacteriana en el hilo de sutura de seda negra post extracción dental simple.

\begin{tabular}{lcc}
\hline & Seda & Control \\
\hline Media & 87,5 & 1,5 \\
D. S. & 206,0 & 1,6 \\
& & \\
Mann-Whitney & 11,000 & \\
Z & $-4,999$ & \\
p & 0,000 & \\
\hline
\end{tabular}

Tabla III. Adhesión bacteriana en el hilo de sutura de nylon post extracción dental simple.

\begin{tabular}{lcr}
\hline & Nylon & Control \\
\hline Media & 99,2 & 1,1 \\
D. S. & 236,1 & 1,0 \\
Mann-Whitney & 33,000 & \\
Z & $-4,349$ & \\
$\mathrm{p}$ & 0,000 & \\
\hline
\end{tabular}

\section{DISCUSIÓN}

Los materiales de sutura se comportan como cuerpos extraños en la herida, induciendo una reacción inflamatoria, debido a los efectos capilares resultantes de la acción de los microorganismos que se adhieren en las heridas. El grado de esta reacción varía según el tipo de sutura (Obermeier et al., 2014) (Polikkotil \& Nath, 2012); además las bacterias se adhieren con diferente afinidad a diversos tipos de materiales de sutura (Banche et al.).

La adhesión bacteriana puede contribuir a la selección de sutura en un paciente propenso a la infección o para el uso de sutura en otras áreas del cuerpo en mayor riesgo de contaminación (Masini et al., 2011); lo que aporta al criterio clínico al momento de planificar la cirugía oral (Javed et al., 2012).

Se puede especular que la mayor adhesión en el hilo de nylon, hallada en el presente estudio, probablemente se deba a que se requieren más nudos para asegurar su permanencia en cavidad oral, debido a que este material presenta una tendencia a regresar a su estado natural recto, lo que se conoce como propiedad de memoria (Armas Moredo et al.). No se encontraron estudios similares con los que se pueda discutir. 
Gil Santos et al. (2012) recomienda utilizar hilos con escasa memoria, debido a que son más manejables y más flexibles. Esta memoria está en relación inversa al diámetro del hilo: a mayor diámetro, menor flexibilidad. Los hilos de sutura deben tener, de ser posible, el menor diámetro con la mayor resistencia tensil y estar concebidos para soportar tensiones hasta que se complete la cicatrización del tejido suturado.

Los resultados obtenidos permiten concluir que:

La adhesión bacteriana fue mayor en el hilo de sutura de nylon comparado con el hilo de sutura de seda negra.

La adhesión bacteriana fue mayor en los hilos de sutura de seda y nylon al compararlos con sus correspondientes controles.

Por los resultados, entre los dos tipos de hilo estudiados, se puede recomendar usar del hilo de seda negra, debido a su menor adhesión bacteriana.

ASMAT-ABANTO, A. S.; ÁVILA-RODRÍGUEZ, A. F. \& MEJÍA-DELGADO, E. Bacterial adhesion in black silk suture thread and nylon post simple dental extraction: controlled clinical trial. Int. J. Odontostomat., 13(2):132-136, 2019.

ABSTRACT: The objective of the study was to compare black silk suture threads and nylon suture with respect to bacterial adhesion after simple dental extraction of posterior pieces. The present study was developed in the Clínica Estomatológica and the Laboratorio de Microbiología of the Unidad de Ciencias Biomédicas y Biotecnología de la Universidad Privada Antenor Orrego (Trujillo, Peru), between August and September 2014. We worked with 19 units sampling for black silk thread and 19 for nylon. The procedure of exodontia for all patients was standardized. After the extraction, the surgical wound was sutured, placing two circumferential points, one with each type of thread. After 7 days, the threads were removed and subjected to microbiological analysis. The bacterial adhesion of the black silk and nylon suture strands was compared using the Wilcoxon test. In addition, the bacterial adhesion of each strand was compared with the adhesion in sterile strands (control) using the Mann-Whitney test for $p<0.005$. When comparing the bacterial adhesion of both threads, a difference was found between them $(p=0.027)$, corresponding greater adhesion to the nylon thread. Bacterial adhesion was higher in nylon suture compared to black silk suture.

KEY WORDS: sutures, tooth extraction, bacterial adhesion.

\section{REFERENCIAS BIBLIOGRÁFICAS}

Altiparmak, N. \& Uckan, S. Bone anchoring of sutures for adequate repositioning of the soft tissue flaps in one stage dental implants. Int. J. Oral Maxillofac. Surg., 42(4):535-6, 2013.

Armas Moredo, K.; Armas Pérez, B. A.; Segura Pujal, L.; Márquez Hernández, J. \& Armas Moredo, K. Materiales de sutura quirúrgica. AMC, 13(5), 2009. Disponible en: http://scielo.sld.cu/ scielo.php?script=sci_arttext\&pid=S1025-02552009000500011

Banche, G.; Roana, J.; Mandras, N.; Amasio, M.; Gallesio, C.; Allizond, V.; Angeretti, A.; Tullio, V. \& Cuffini, A. M. Microbial adherence on various intraoral suture materials in patients undergoing dental surgery. J. Oral Maxillofac. Surg., 65(8):1503-7, 2007.

Bin AIShaibah, W. M.; El-Shehaby, F. A.; El-Dokky, N. A. \& Reda, A. R. Comparative study on the microbial adhesion to preveneered and stainless steel crowns. J. Indian Soc. Pedod. Prev. Dent., 30(3):206-11, 2012.

Burkhardt, R.; Preiss, A.; Joss, A. \& Lang, N. P. Influence of suture tension to the tearing characteristics of the soft tissues: an in vitro experiment. Clin. Oral Implants Res., 19(3):314-9, 2008.

Cruz, F.; Leite, F.; Cruz, G.; Cruz, S.; Reis, J.; Pierce, M. \& Cruz, M. Sutures coated with antiseptic pomade to prevent bacterial colonization: a randomized clinical trial. Oral Surg. Oral Med. Oral Pathol. Oral Radiol., 116(2):e103-9, 2013.

Edmiston, C. E.; Seabrook, G. R.; Goheen, M. P.; Krepel, C. J.; Johnson, C. P.; Lewis, B. D.; Brown, K. R. \& Towne, J. B. Bacterial adherence to surgical sutures: can antibacterial-coated sutures reduce the risk of microbial contamination?. J. Am. Coll. Surg., 203(4):481-9, 2006.

Gay, C. \& Berini, L. Tratado de Cirugía Bucal. Barcelona, Océano, 2006.

Ghoreishian, M.; Gheisari, R. \& Fayazi, M. Tissue adhesive and suturing for closure of the surgical wound after removal of impacted mandibular third molars: a comparative study. Oral Surg. Oral Med. Oral Pathol. Oral Radiol. Endod., 108(1):e14-6, 2009.

Gil Santos, L.; Más-Estellés, J.; Salmerón Sánchez, M. \& Barrios, C. Comportamiento mecánico de 3 tipos de anudados quirúrgicos usando monofilamentos de 4/0. Cir. Esp., 90(6):388-93, 2012.

Israr, M. \& Stassen, L. F. The comparison of scalp closure with staples, silk, prolene and vicryl following a Gillie's temporal approach for malar / zygomatic complex fracture; a prospective study. Pak. Oral Dent. J., 33(1):3-7, 2013.

Javed, F.; Al-Askar, M.; Almas, K.; Romanos, G. E. \& Al-Hezaimi, K. Tissue reactions to various suture materials used in oral surgical interventions. ISRN Dent., 2012:762095, 2012.

Lüdecke, C.; Jandt, K. D.; Siegismund, D.; Kujau, M. J.; Zang, E.; Rettenmayr, M.; Bossert, J. \& Roth, M. Reproducible biofilm cultivation of chemostat-grown Escherichia coli and investigation of bacterial adhesion on biomaterials using a non-constant-depth film fermenter. Plos One, 9(1):e84837, 2014.

Masini, B. D.; Stinner, D. J.; Waterman, S. M. \& Wenke, J. C. Bacterial adherence to high--tensile strength sutures. Arthroscopy, 27(6):834-8, 2011.

Nazar, C. J. Biofilms bacterianos. Rev. Otorrinolaringol. Cir. Cabeza Cuello, 67(1):161-72, 2007.

Nozaki, K.; Mori, R.; Ryoke, K. \& Uchio, Y. Comparison of elastic versus rigid suture material for peripheral sutures in tendon repair. Clin. Biomech. (Bristol, Avon), 27(5):506-10, 2012.

Obermeier, A.; Schneider, J.; Wehner, S.; Matl, F. D.; Schieker, M.; von Eisenhart-Rothe, R.; Stemberger, A. \& Burgkart, R. Novel high efficient coatings for anti-microbial surgical sutures using chlorhexidine in fatty acid slow-release carrier systems. PloS One, 9(7):e101426, 2014. 
Pons-Vicente, O.; López-Jiménez, L; Sánchez-Garcés, M. A.; SalaPérez, S. \& Gay-Escoda, C. A comparative study between two different suture materials in oral implantology. Clin. Oral Implants Res., 22(3):282-8, 2011.

Venema, S.; Abbas, F.; van de Belt-Gritter, B.; van de Mei, H. C.; Busscher, H. J. \& van Hoogmoed, C. G. In vitro oral biofilm formation on triclosan-coated sutures in the absence and presence of additional antiplaque treatment. J. Oral Maxillofac. Surg., 69(4):980-5, 2011.

Wong, V. W.; Rao, S. K. \& Lam, D. S. Polyglactin sutures versus nylon sutures for suturing of conjunctival autograft in pterygium surgery: a randomized, controlled trial. Acta Ophthalmol. Scand., 85(6):658-61, 2007

Zijnge, V.; van Leeuwen, M. B.; Degener, J. E.; Abbas, F.; Thurnheer, T.; Gmür, R. \& Harmsen, H. J. Oral biofilm architecture on natural teeth. Plos One, 5(2):e9321, 2010.
Dirección para correspondencia:

Angel S. Asmat-Abanto

Docente de la Escuela de Estomatología de la

Universidad Privada Antenor Orrego

Doctorado en la Escuela de Postgrado de la

Universidad Nacional de Trujillo

PERÚ

Email: aasmata1@upao.edu.pe

Recibido : 14-09-2018

Aceptado: 10-01-2019 